\title{
Design of Digital Communication Process for Condition Monitor Machine Tool
}

\author{
Yen-Hsiang Huanga, ${ }^{\mathrm{a},}$, Yun-Hui Liu', Kuo-Shen Chen ${ }^{\mathrm{b}}$ \\ ${ }^{a}$ Department of Mechanical Engineering, Southern Taiwan University of Science and Technology, No.1 Nantai St., \\ Yungkang Dist., Tainan City 710, Taiwan \\ ${ }^{b}$ Department of Mechanical Engineering, National Cheng-Kung University, No.1 Daxue Rd., East Dist., Tainan City \\ 701, Taiwan
}

*Corresponding Author: MA710105@stust.edu.tw

\begin{abstract}
Machine tool monitoring plays an important role in Industry 4.0. Appropriate vibration monitoring would be essential for the safety and life assessment of machine tools. However, currently used piezoelectric or MEMS based accelerometers all have their own sensitivity and bandwidth limitations and analog sensors are also susceptible to environmental noise thus it may not be suitable for machine tool monitoring. On the other hand, digital sensors could have better noise rejection capabilities. However, for properly utilizing the advantage of digital sensors, important issues such as proper setup of communication protocols and sampling frequency optimization must be pre-determined in using digital accelerometers. In this work, a digital MEMS accelerometer device is used to measure the current sampling frequency through the communication protocol under the test environment, and successfully optimized the $60 \%$ sampling speed to give a higher bandwidth measurement, and then calibrated the digital acceleration on the shaker. Finally, Multiple digital accelerometers are integrated. In this way, multiple digital sensors can be integrated in the future, and sensor fusion is performed through a back-end integrated data system, which can be practically used in machine tools monitoring.
\end{abstract}

Keywords: digital MEMS accelerometer, machine tools monitoring.

\section{Introduction}

With the rapid development of global industrial technology, the industry has developed "Industry 4.0 " in order to improve productivity and reduce production costs, convert physical signals into digital signals, establish Internet connection with users and let users know the production status of products. It is an important topic for developing Industry 4.0(1). One of the key technologies is the information of the machine tool. Through purpose of status monitoring and system early warning(2), it will be better understood to achieve machine fault monitoring and diagnosis, life prediction, processing quality inspection, yield control and capacity improvement. The vibration of the machine tool is particularly important for the state of the machine tool because the machine tool vibration source is very complex, such as bearing damage, unbalanced rotating shaft and loose spindle. These vibration sources have different frequency characteristics and are basically distributed within $10 \mathrm{kHz}$. While traditional accelerometers have been used to measure vibration in this area, single accelerometers are limited to sensitivity and bandwidth limitations and are very expensive.

Recently, with the advanced in MEMS (microelectromechanical systems) technology, MEMS accelerometers become an alternative choice in vibration measurement and they have been widely used in automobile and mobile communications. Previously, 2-axis MEMS accelerometers are also used to monitor rotating machine, such as motor(3).The design of 3-axes MEMS accelerometers have also been applied on robot arm(4). However, their performances are usually inferior and any single MEMS accelerometer also suffer from the same sensitivity-bandwidth tradeoff as having seen in piezoelectric accelerometers

Despite of the above concerns, MEMS accelerometers are still attractive due to its nature of low cost and miniaturization design. Previously, an integrated vibration 
sensing module was proposed by integrating various analog MEMS accelerometer chips. Through structure and circuit design, the vibration sensing module were integrated as well as the disadvantage, low sensitivity, of MEMS accelerometer was overcome, and it was actually used for machine tool measurement (5), However, the analog accelerometer has the following disadvantages. The analog accelerometer signal is easy to absorb environmental noise, which will affect the minimum sensitivity that the sensor can measure, which reduces its performance. In addition to the current development of monitoring systems, various sensors need to be digitally integrated. However, analog sensors need to be equipped with ADC (Analog-to-digital converter) and complex data conversion. It is more difficult to integrate the monitoring system. It is more difficult to integrate the monitoring system. Through the above points, digital sensors could have better noise rejection capabilities. However, for properly utilizing the advantage of digital sensors, important issues such as proper setup of communication protocols and sampling frequency optimization must be pre-determined in using digital accelerometers.

Motivated by the above needs, the overall goal is to design a program to integrating digital accelerometers for testing and experiments. Technically, there are three steps to achieve the final goal. First, the overall system should be designed. Second, the digital MEMS accelerometer should be calibrated and tested, and the transmission protocol and sampling rate are adjusted. Finally, several digital MEMS accelerometers must be integrated for testing the overall performance.

In this work, we mainly present the efforts on digital MEMS accelerometers integration and communication protocol for signal processing. The overall scope of this work is shown in Fig.1. Two types of MEMS accelerometers with different frequency response characteristics are chosen for performing this study

Meanwhile, In order to ensure that the signal processing can completely capture the original digital signal, the system sampling frequency is optimized. The initial demonstration of the integrated MEMS accelerometer will be integrated with the KX222, KX124 three-axis digital accelerometer and tested against Shaker by I2C protocol.

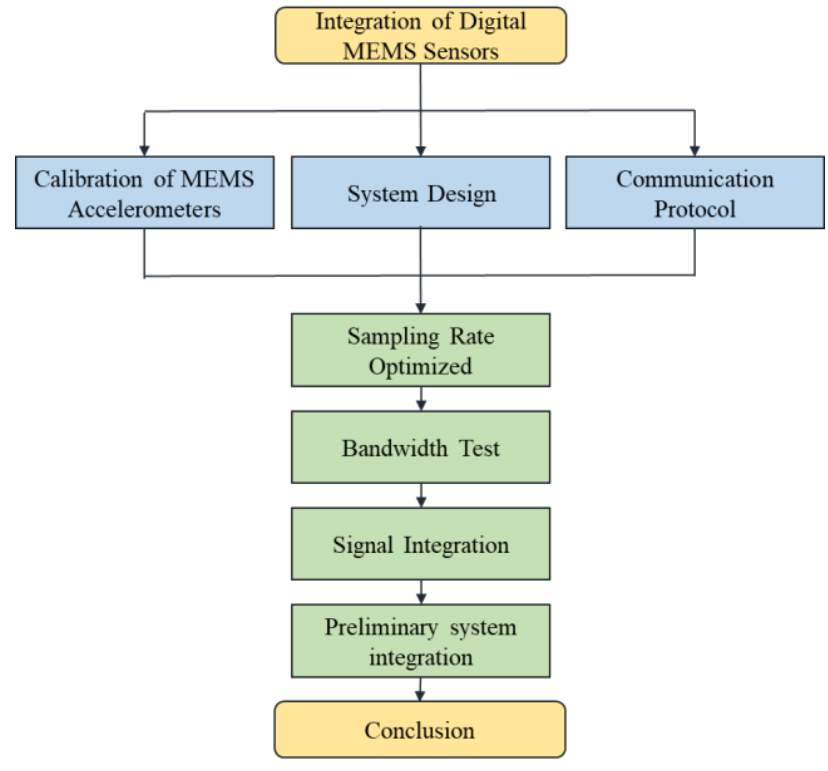

Fig. 1. The overall scope of this paper.

\section{Design and Analysis}

\subsection{System Design}

The system architecture diagram is shown in Fig. 2. The main target is to establish a system by using LabVIEW and myRIO to integrate two types of digital MEMS accelerometers signals. In order to facilitate the integration of multiple digital sensors. before achieving the final objective, several tasks should be finished. First, select the appropriate digital MEMS accelerometer and understand it's communication protocol. Second, reading vibration signal whether the program as scheduled and calibrating the digital MEMS accelerometers. Finally, integrating two type of digital MEMS accelerometers signal. More details are highlighted below.

\subsection{Selection of Digital MEMS Accelerometers}

There are two types of digital MEMS accelerometers selected due to the different dynamic characteristics of the machine tool, In order to detect high frequency vibration range, the Kionix KX222 tri-axial accelerometer is chosen because of it's wider bandwidth (up to $8 \mathrm{kHz}$ ) 、 larger measurement range ( $\pm 32 \mathrm{~g})$ and higher Resolution(16Bits). Meanwhile, in order to detect vibration in middle and low frequency range, the tri-axial $\mathrm{KX} 124$ is chosen. The bandwidth of this sensor can be up to $3.5 \mathrm{kHz}$ and good resolution (16 Bits) in the middle and low frequency range sensors. These two digital accelerometers support both Serial Peripheral Interface (SPI) and Inter-Integrated Circuit(I2C) 
protocols. We have these two types of sensors for calibration test and integrate experimental test.

\subsection{Communication Protocol}

As stated above, the digital accelerometers that we chosen has two communication protocols, SPI and I2C. Both of them can integrate multiple digital sensors at the same time. The main difference is that the $\mathrm{I} 2 \mathrm{C}$ interface can integrate multiple sensors with only 2 wires, as shown as Fig.3.But the Sampling rate is only $400 \mathrm{kHz}$.Then the SPI interface base requires 3 wires, the wire of the communication is calculated by.

$$
W=N+3
$$

Where $\mathrm{N}$ is the number of integrated sensors. It interface shown in Fig.4.But the Sampling rate is faster than I2C(up to $4 \mathrm{MHz}$ ). In order to reduce the wire of the communication, we chose the $\mathrm{I} 2 \mathrm{C}$ protocol for sensors communication and integration.

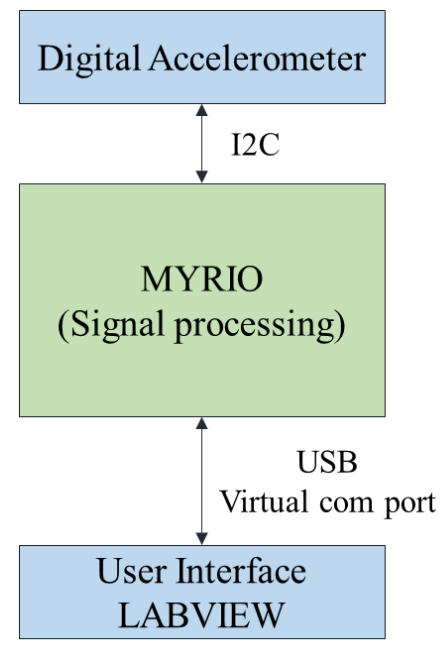

Fig. 2 System Architecture Diagram

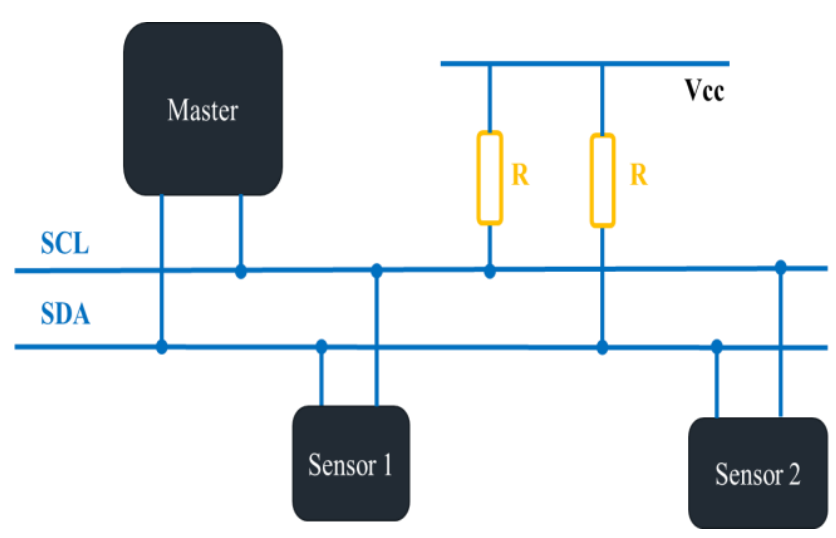

Fig. 3 I2C Interface Diagram

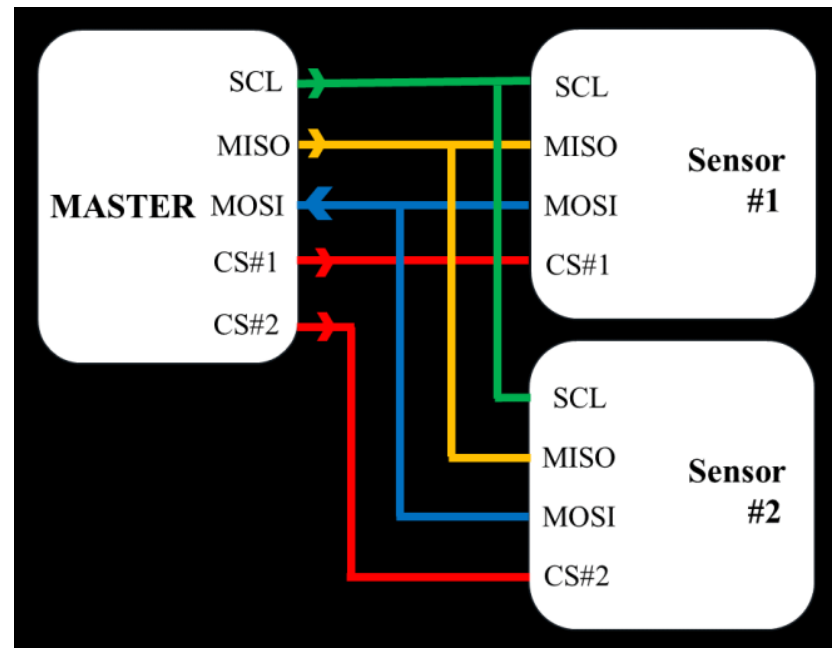

Fig. 4 SPI Interface Diagram

\section{Testing system setup}

\subsection{Intelligent Data System Test}

Intelligent Data System is written in LABVIEW. The program flow is shown in Fig. 5. First, the parameters of the communication protocol must be set. Second, Sampling the data. Finally, the data was output to Fast Fourier Transform( FFT). Because accelerometers require a higher sampling frequency, and the normal sampling method will cause the sampling frequency to be seriously insufficient, so the sampling program was optimized. When the original communication protocol reads the data, it needs to call its address for data acquisition. It significantly slows down the speed. The comparison before and after the optimization of the process is shown in Fig.6. The original sampling program takes the fastest $0.146 \mathrm{mS}$ to read 16Bits data. The maximum sampling frequency after conversion is only $6850 \mathrm{~Hz}$. The fastest is $0.088 \mathrm{mS}$, the maximum sampling frequency after conversion is $11290 \mathrm{~Hz}$, and the sampling part is optimized for $60 \%$ speed.

\subsection{Digital MEMS Accelerometers Bandwidth Test}

The accelerometer calibration system is shown in Fig.7. MEMS accelerometers such as KX222 and KX124 are fixed on the adapter, while the piezoelectric accelerometer (Endevco, 752A13) served as the reference standard is also fixed on the same reference surface, and its characteristics is shown in Table.1. The shaker (TIRA, TV51120) outputs a sweep signal, which is measured by both accelerometers, the piezoelectric accelerometer signals are acquired by a spectrum analyzer (B\&K type $3560 \mathrm{C}$ ) and the MEMS accelerometers signals are acquired by MYRIO for 
computing their frequency characteristics, Then divide the two calculated spectral characteristics to obtain Frequency Response Function(FRF). The results are shown in Fig.8(a) (b)(c). The bandwidth of $\mathrm{KX} 124$ is $1800 \mathrm{~Hz}$ in z-axis, $3700 \mathrm{~Hz}$ in $\mathrm{x}$ and $\mathrm{y}$-axis under a supplied power of $3.3 \mathrm{~V}$, which is similar to the vendor's data. Meanwhile, KX222 is measured by the same setup. Because the sampling of the program can only up to $11 \mathrm{kHz}$, the measurement bandwidth can only reach $5 \mathrm{kHz}$. The measurement results are shown in Fig. 8-9(d)(e)(f), the bandwidths in $\mathrm{x} y$ and $\mathrm{z}$-axis are smooth to $5 \mathrm{kHz}$.

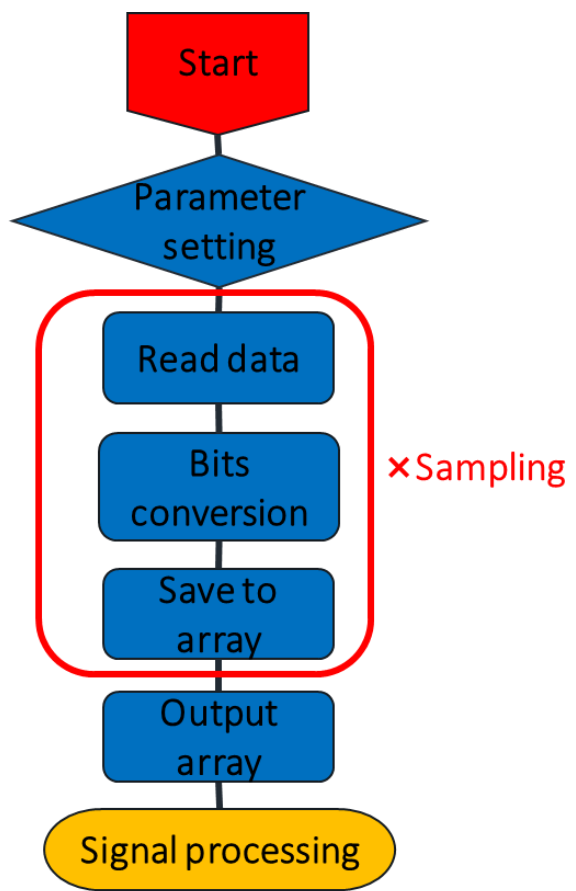

Fig. 5 The program flow Diagram

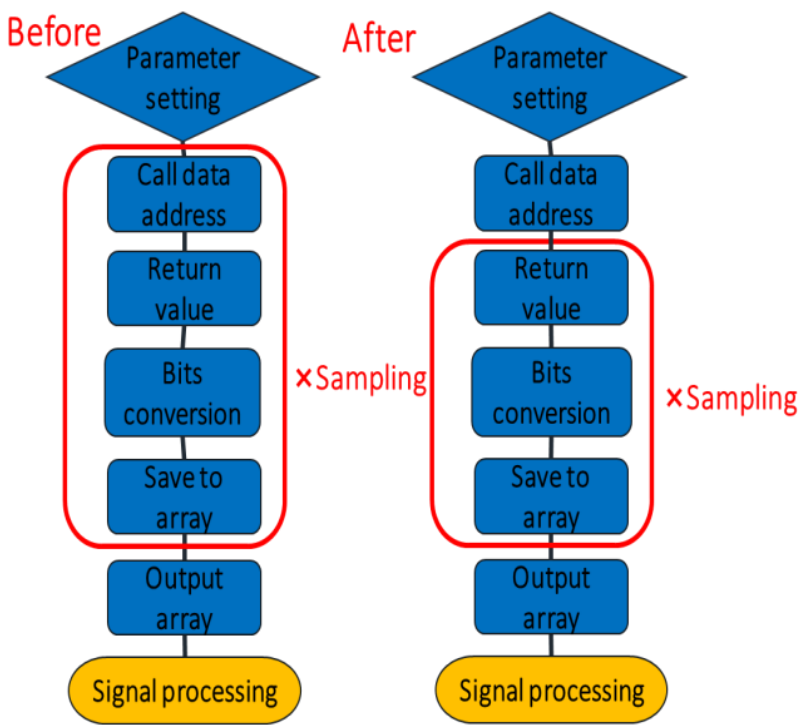

Fig. 6 The comparison before and after the optimization of the process

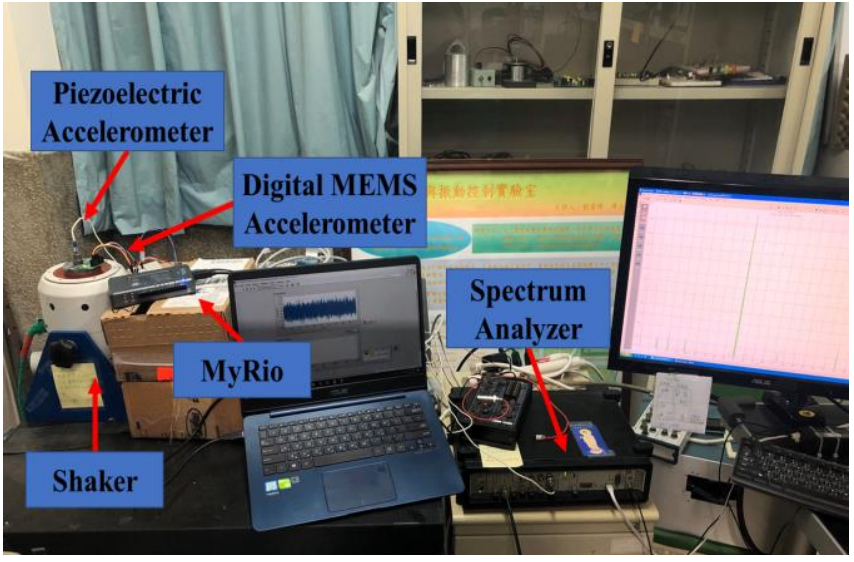

Fig. 7 The accelerometer calibration system
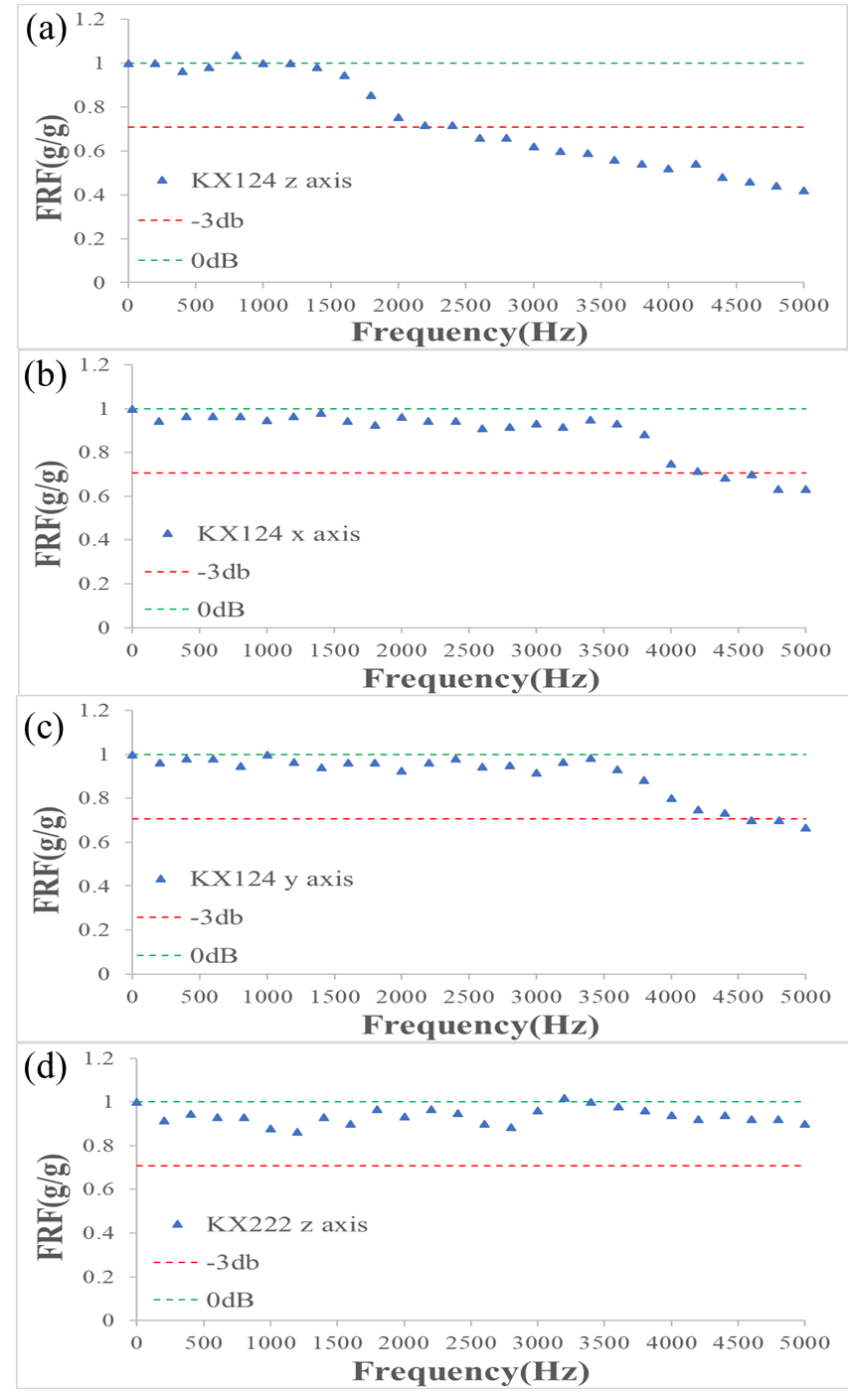

Fig. 8 The Frequency Response Function of(a) the $\mathrm{z}$ axis of KX124(b) the $x$ axis of KX124(c) the $y$ axis of KX124(d) the $\mathrm{z}$ axis of $\mathrm{KX} 222$ 

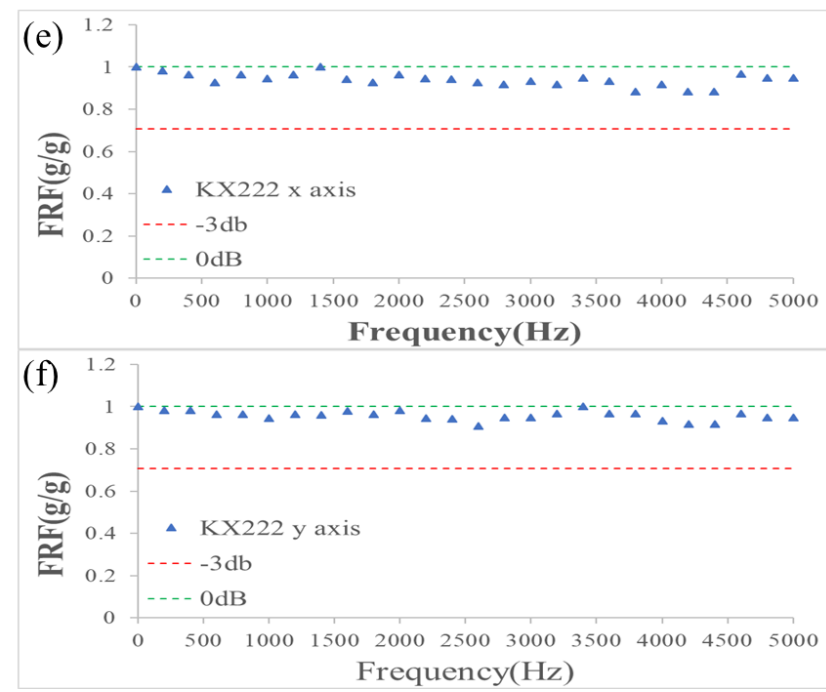

Fig. 9 The Frequency Response Function of (e) the $\mathrm{x}$ axis of KX222(f) the y axis of KX222

Table 1. The characteristics of Endevco 752A13 accelerometers

\begin{tabular}{|c|c|c|}
\hline Model Property & Sensitivity(mV/g) & Bandwidth(Hz) \\
\hline ENDEVCO 752A13 & 1000 & 10000 \\
\hline
\end{tabular}

\section{System Integration}

In this section, the MEMS accelerometer, KX124 and KX222, are used for measuring the vibration signal of the fixed frequency generated by the shaker, and then the signal will be transmitted back to the user interface in real time through the communication protocol as the first step toward the digital signal integration. The experimental architecture is shown in Fig. 10. The user interface is designed to confirm the overall system feasibility through the following methods. Instantly display the time domain signal and the spectrum analysis result through the user interface for immediate display to observe whether it is consistent with the vibration frequency generated by the shaker. From Fig. 11, it is observed that the $200 \mathrm{~Hz}$ vibration signals generated by shaker can be correctly discriminated by the intelligent data system after the raw data recording and the online analysis through the user interface

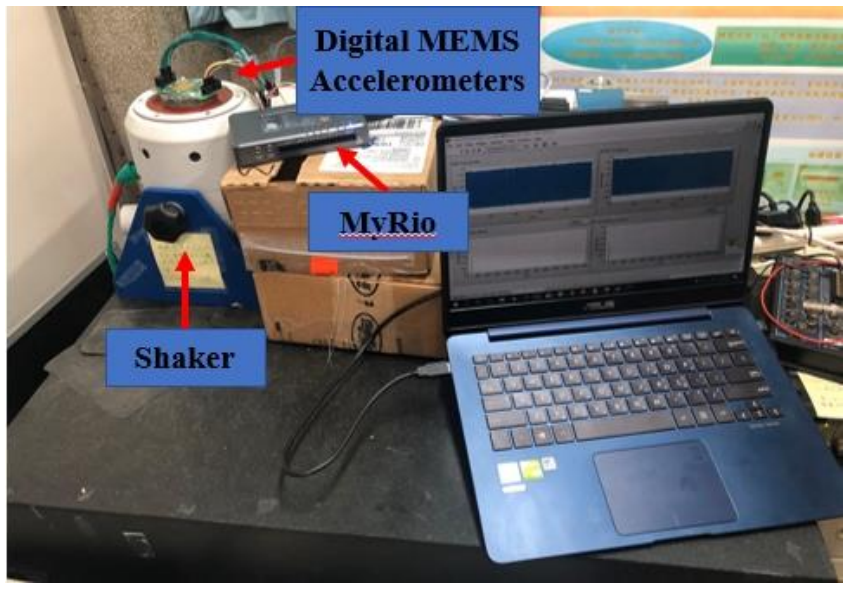

Fig. 10 The experimental architecture

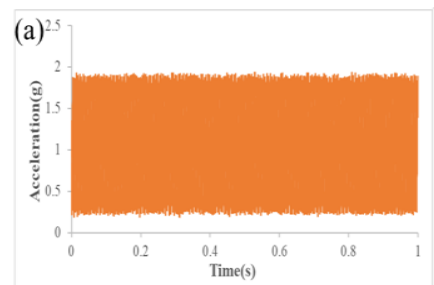

(c) 2.5
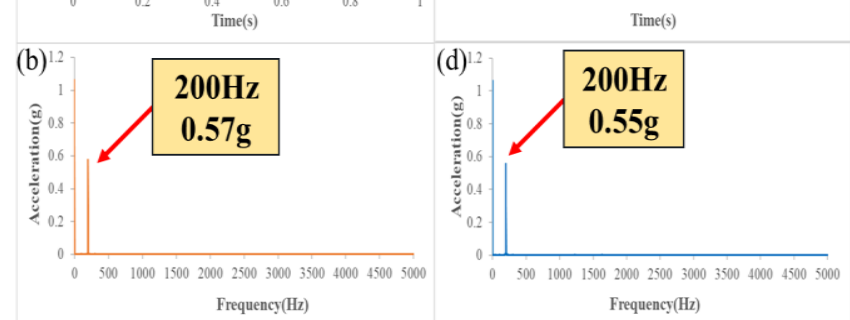

Fig. 11 Shaker $200 \mathrm{~Hz}(\mathrm{a}) \mathrm{KX} 124$ time domain(b)KX124 frequency domain(c)KX222 time domain(d)KX222 frequency domain

\section{Preliminary system integration}

At present, An adaptor for integrating digital MEMS sensors has been preliminary designed. As shown in Fig.12, the sensor is expected to be placed on the PCB (Printed circuit board). There is a threaded hole at the bottom of the adaptor for easy removal. The shell cover has the effect of isolating noise. The actual finished product will change according to the measurement environment. Our system architecture will be shown in Fig.13, which will integrate digital MEMS sensors, use MYRIO to do sampling and signal processing and upload to the cloud, and watch the characteristic signals from the HMI (human-machine interface). In the future, this architecture will be applied to machine tools for measurement. 


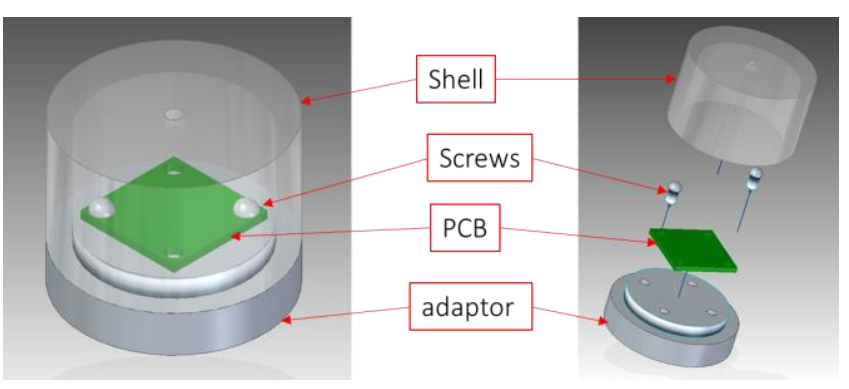

Fig. 12 the design diagram of the integrated module

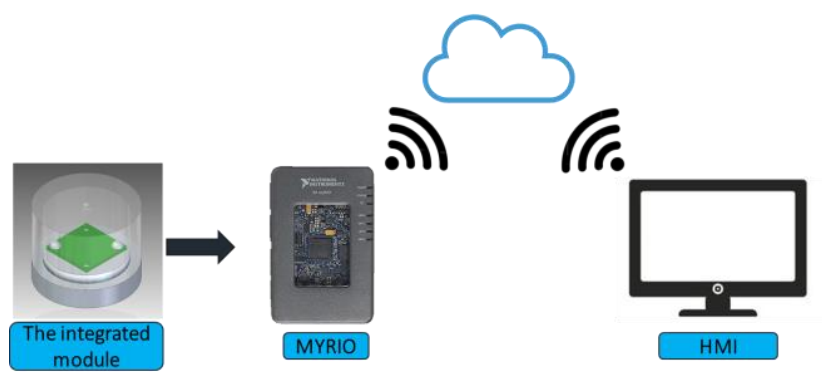

Fig. 13 the system architecture

\section{Conclusion and future works}

This article mainly presents the design and initial test of data reading system integrated with digital MEMS accelerometer. By such an approach, it is desirable to integrate multiple digital MEMS accelerometers. Currently, the dynamic characteristics of all selected digital MEMS accelerometers have been obtained through the design of the data reading system. and a variety of digital MEMS accelerometers have been integrated through the communication protocol. From the experimental results, it can be found that the intelligent data system proposed by this study can correctly sample the vibration raw data information and correctly analyze the spectrum through the FFT algorithm to integrate multiple digital accelerometers. In the near future, in addition to optimize the effort of the sampling frequency, to these two accelerometers and other sensors would be integrated into the module, also provides a more complex designs in the transport protocol and signal processing, to provide better Dynamic characteristics.

\section{Acknowledgment}

This research is supported by the Ministry of Science and Technology (MOST) in Taiwan, ROC under the contract numbers 106-2221-E-218-014, 107-2221-E-218-029.

\section{References}

(1) M. Rüßmann, M. Lorenz, P. Gerbert, M. Waldner, J.
Justus, P. Engel, and M. Harnisch, "Industry 4.0: The Future of Productivity and Growth in Manufacturing Industries,” Boston Consulting Group, 2015

(2) D. Ni, M. Jing, H. Fan, M. Li, H. Liu, J. Li, "Study on monitoring and warning system for high-speed motorized spindle based on vibration signals", Proc. 2013 Fifth Conference on Measuring Technology and Mechatronics Automation, pp. 1076-1079, 2013

(3) S. B. Chaudhury, M. Sengupta, K. Mukherjee : "Vibration Monitoring of Rotating Machines Using MEMS Accelerometer", International Journal of Scientific Engineering and Research, Vol. 2, pp. 5-11, 2014.

(4) A. A. Jaber and R. Bicker : "Design of a Wireless Sensor Node for Vibration Monitoring of Industrial Machinery," International Journal of Electrical and Computer Engineering, Vol. 6, pp. 639-653, 2016.

(5) I. C. Sun, J. M. Tsai and K. S. Chen, "Design and realization of a sensing module for machine tools status monitoring by multiple MEMS accelerometers integration," Taiwan Precision Technology Workshop, 2018 